J Neurosurg Anesthesiol. 2016 October ; 28(4): 361-372. doi:10.1097/ANA.0000000000000348.

\title{
A Systematic Review of Pre-clinical Studies
}

\author{
William M. Jackson, MD¹, Christy D.B. Gray, MD, $\mathrm{PhD}^{2}$, Danye Jiang, BS ${ }^{2}$, Michele L. \\ Schaefer, $\mathrm{PhD}^{2}$, Caroline Connor, MD, $\mathrm{PhD}^{2}$, and C. David Mintz, MD, PhD $^{2}$ \\ ${ }^{1}$ Department of Anesthesiology, Columbia University College of Physicians and Surgeons, New \\ York, NY 10032 \\ 2 Department of Anesthesiology and Critical Care Medicine, The Johns Hopkins University School \\ of Medicine, Baltimore, MD, USA
}

\begin{abstract}
Data from epidemiologic studies and animal models have raised a concern that exposure to anesthetic agents during early postnatal life may cause lasting impairments in cognitive function. It is hypothesized that this is due to disruptions in brain development, but the mechanism underlying this toxic effect remains unknown. Ongoing research, particularly in rodents, has begun to address this question. In this review we examine currently postulated molecular mechanisms of anesthetic toxicity in the developing brain, including effects on cell death pathways, growth factor signaling systems, NMDA and GABA receptors, mitochondria, and epigenetic factors. The level of evidence for each putative mechanism is critically evaluated, and we attempt to draw connections between them where it is possible to do so. While there are many promising avenues of research, at this time no consensus can be reached as to a definitive mechanism of injury.
\end{abstract}

\section{Introduction}

Although the modern practice of pediatric anesthesia is remarkably safe in terms of mortality and gross morbidity, there is accumulating evidence that exposure to anesthetic agents may cause changes in brain development that ultimately result in permanent deficits in cognitive function. This phenomenon, dubbed pediatric anesthetic neurotoxicity (PAN), has been widely debated in the academic anesthesiology and surgical literature, including occasional pieces in high profile journals ${ }^{1}$, and it has been echoed in the lay press as well. The United States Food and Drug Administration (FDA) has identified PAN as a potentially important public health problem ${ }^{2}$, and is conducting intramural research as well as supporting extramural research through a public-private partnership known as Smart-Tots ${ }^{3}$, and via a request for grant applications entitled the Pediatric Anesthesia Safety Initiative. Currently, the key research questions in PAN include the following:

1. Do meaningful cognitive deficits occur in human pediatric patients exposed to anesthetics?

Correspondence can be addressed to: C. David Mintz, MD, PhD, Department of Anesthesiology and Critical Care Medicine, Ross 370, 720 Rutland Ave, Johns Hopkins Medical Institutes, Baltimore, MD 21205, Phone: 917-733-0422, cmintz2@jhmi.edu. No conflicts of interest exist for any authors listed above. 
2. If so, under what conditions does this occur?

3. Can deficits due to anesthetic exposure be mitigated or avoided?

Large prospective, randomized human trials that could definitively answer these questions are difficult to design and execute, due both to ethical and practical concerns. While several trials are underway, and they were discussed extensively at the Fifth Pediatric Anesthetic Neurotoxicity Developmental Assessment Symposium, the results of several of these studies are many years away. Even if all the currently envisioned studies are carried out successfully, many questions will remain unanswered.

The strongest evidence for PAN is the confluence of two distinct and relatively well-evolved lines of research: retrospective epidemiologic studies of human patient cohorts and preclinical investigations in animal models. Epidemiologic studies have shown correlations between exposure to surgery and anesthesia and subsequent reductions in performance in school testing, increases in billing codes relating to behavioral and learning disorders, and deficits in cognitive testing ${ }^{4-8}$. These studies are open to a range of interpretations due to inevitable confounding factors such as surgery and co-morbid disease, but when taken in the context of the preclinical literature in animal models they are highly suggestive. Since the initial observation by Ikonomidou and coworkers ${ }^{9}$, different groups ${ }^{10-14}$ have confirmed that early postnatal exposure to anesthetics results in long-lasting impairments in learning and memory. These studies have been conducted primarily in rodents, but newly emerging data in non-human primates suggests that developmental anesthesia exposure causes changes in brain function across species ${ }^{15-17}$. However, data from animal models can do more for the study of PAN than just support the hypothesis that developmental anesthetic exposure can lead to neurobehavioral changes.

At present, the principal value of animal model research is to uncover the mechanism of injury of anesthetic neurotoxicity in PAN. A clear understanding of how anesthetics administered during development can have a lasting impact on brain function will both speak to the plausibility of the phenomenon and also provide valuable insights on how it might be prevented. In this review, we will examine cellular and molecular mechanisms of injury that have been proposed in PAN in order to evaluate the quality of the evidence and to look for common themes or connections that will move the field toward a cohesive model of developmental anesthetic neurotoxicity.

\section{Cell Death Pathways}

The first potential mechanism of developmental anesthetic toxicity to be proposed was apoptotic cell death ${ }^{10}$, and the importance of cell death in models of PAN remains a subject of ongoing debate and active investigation ${ }^{18}$. Apoptosis is a sequence of programmed cell death that is conserved across a wide variety of eukaryotes ${ }^{19}$. Across animal species, apoptosis is an important mechanism during development that is designed to remove superfluous cells and form the organs of the body, and it plays a critical role in normal brain development ${ }^{20}$. It is also a cellular response to a variety of stresses, such as hypoxia ${ }^{21}$, reactive oxygen species ${ }^{22}$, increased intracellular calcium ${ }^{23}$, viral infection ${ }^{24}$, radiation ${ }^{25}$, and exposure to toxins ${ }^{26}$. Unlike cell necrosis, which is an unorganized form of cell death, 
apoptosis results from a signaling cascade initiated in one of two ways. The intrinsic pathway involves intracellular activation of proteins from the Bcl-2 family. This family contains both pro- and anti-apoptotic proteins whose balance is tightly regulated. When cellular stresses disrupt this balance, pro-apoptotic Bax and Bak form permeability pores on the mitochondrial membrane that allow the release of cytochrome $\mathrm{c}$, the rate-limiting step in the initiation of apoptosis. Once cytochrome $\mathrm{c}$ is released into the cytosol, initiator caspase-9 is activated by apoptotic protease activating factor-1, or APAF. Caspase 9 activates the final effector caspases 3,6 , and 7, which formally initiate apoptosis ${ }^{27}$. The extrinsic pathway of apoptosis is initiated through external signaling molecules that bind receptors on the cell surface. The best characterized initiator receptors are the tumor necrosis factor- $a$ (TNF- $a$ ) receptor and the Fas receptor ${ }^{28}$. The pathway begins with the extracellular signals TNF-a and FasL activating their respective receptors, which contain intracellular death domains, which in turn activate caspase- 8 and caspase-3, committing the cell to apoptosis in a common final pathway shared with the intrinsic path. A schematic diagram of these apoptotic pathways is shown in Figure 1.

Hence anesthetics could act on a wide array of targets that initiate the pathway of apoptosis. Ikonomidou and coworkers ${ }^{9}$ first reported the possible interaction of apoptotic pathways with common anesthetic targets. They showed an association between administration of $\mathrm{N}$ methyl-D-aspartate (NMDA) receptor antagonists, including the common anesthetic drug ketamine, and widespread neuronal apoptosis in the cortex, thalamus, hypothalamus, and hippocampus as assessed by TUNEL staining in rats exposed during days of life 1-14. The study was well controlled and ruled out several other hypothesized mechanisms of neural apoptosis, by inducing blockade of calcium channels, AMPA glutamate receptors, muscarinic receptors, and dopamine receptors. None had the same widespread induction of apoptosis as the three NMDA receptor antagonists used in the study.

This work led to the study of other drugs that inhibit NMDA receptors, such as volatile anesthetics ${ }^{29}$, nitrous oxide ${ }^{30}$, ethanol ${ }^{30}$, and xenon ${ }^{30}$. Ethanol, a combined NMDA antagonist/gamma-Aminobutyric acid receptor subtype $\mathrm{A}\left(\mathrm{GABA}_{\mathrm{A}}\right)$ potentiator, was associated with a similar widespread neuronal degeneration to MK-801. In addition, this work tested a variety of GABAergic drugs, such as phenobarbital, pentobarbital, diazepam, and clonazepam, all of which induced neuroapoptosis by TUNEL staining in different thalamic nuclei and different layers of the cerebral cortex ${ }^{31}$. Ethanol, having both effects, had a qualitative spatial distribution somewhere between the two more specific compounds. Thus, multiple drugs commonly used for anesthesia have actions that converge into the final common pathway of programmed cell death. How exactly these compounds caused such a profound change in cellular architecture requires further study.

Jevtovic-Todorovic further bolstered the case for an apoptotic mechanism when they exposed 7-day-old rats to a combination of midazolam, nitrous oxide, and isoflurane for 6 hours ${ }^{10}$. This triple combination caused a widespread neural degeneration similar to that observed in previous studies; however, the slices were immunolabeled for activated caspase-3, which was present in a similar distribution as the degenerative cells, suggesting the activation of apoptotic pathways. This finding has since been confirmed in several other studies in rats ${ }^{32}$, guinea pigs ${ }^{33}$, and macaques ${ }^{13,15,16}$ using a variety of different markers of 
apoptotic cell death. It has been extended to sevoflurane ${ }^{34,35}$, as well as propofol, another commonly used anesthetic agent ${ }^{36-39}$. In summation, exposure of a variety of experimental animals and cell cultures to anesthetics with GABA agonist and NMDA antagonist actions during neuron and glial cell development is associated with widespread apoptosis of both neurons and glia in a variety of brain structures.

However, the exact mechanism by which these drugs induce neural degeneration remains incompletely understood. Investigators have tried to elucidate which apoptotic pathway was activated prior to apoptosis. Yon found that, by replicating the "triple cocktail" of midazolam, nitrous oxide, and isoflurane, first the intrinsic and then the extrinsic pathway was activated after several hours of exposure ${ }^{11}$. They found an increase in levels of cytochrome c, caspase-3, -8 , and -9 , and a decrease in levels of anti-apoptotic protein Bcl-X, implying activation of both systems. Furthermore, they found that these changes occurred in rats on postnatal day 7 . Interestingly, on post-natal day 14 proteins levels changed in the opposite direction from postnatal day 7 , implying an activation of a neuroprotective mechanism at this time point. The exact target of these anesthetics that led to changes in apoptotic pathway activity was not explored in these studies. Zhang found evidence in vitro of an association of isoflurane anesthesia and an increase in cytosolic calcium concentration $^{40}$, and Kahraman found a similar trend in association with propofol ${ }^{14}$. Increases in calcium ion concentration could be mediated through depolarizing actions of GABA in developing neurons undergoing synaptogenesis ${ }^{41}$ which could account for the absence of this effect later in development, but this mechanism has not yet been fully explored.

Not all studies of anesthetics delivered during development show activation of apoptotic pathways. Nyman et al. found that etomidate, which acts on GABA receptors like many of the agents discussed above, does not induce apoptosis when given in a single low-, medium-, or high-dose regimen on PND10 in rats ${ }^{42}$. Another work found that short exposure to lowdose sevoflurane for only 3 hours resulted in no significant increase in apoptosis ${ }^{43}$. This may imply a dose threshold phenomenon of neuroapoptosis, and it does raise the issue of whether doses used in pediatric anesthesia are sufficient to induce apoptosis. Interestingly, xenon, a noble gas anesthetic that antagonizes NMDA receptors, was found to inhibit the apoptosis induced by isoflurane in PND7 rat cortex and hippocampus in a dose-dependent manner ${ }^{12}$. This complicates the picture as ketamine induces, and in combination with isoflurane, worsens neuronal apoptosis, yet putatively acts through a similar mechanism. Also, no study has yet addressed the possibility that enhanced apoptosis is an epiphenomenon of anesthetic toxicity rather than an actual cause of cognitive changes. It may be that apoptosis is simply a consequence of disruptions in connectivity, and in that setting it might even be beneficial, as developmental apoptosis is thought to serve to eliminate spurious circuitry.

In spite of the concerns discussed above, the overall quality of the data for rats and neuronal apoptosis is quite robust, and an increase in apoptosis remains the best studied putative mechanism for PAN. Most of the experiments are well-designed and generally adhere to ARRIVE guidelines ${ }^{44}$. They are perhaps hampered by low sample size and variations in technique, but the repeated and consistent findings point to a likely association. Data in primates is somewhat smaller in volume and seems to qualitatively involve a less significant 
insult in terms of apoptotic cells per unit volume. It remains unclear how the relevance of apoptosis can be translated to humans, as there is no non-invasive in vivo modeling of human neuronal apoptosis, but this is a limitation common to other mechanisms as well.

\section{Growth Factor Signaling Systems}

Growth factors are critical for neuronal development and survival. A family of growth factors called neurotrophins has been identified as critical to the processes of cell survival, synaptogenesis, and hippocampal plasticity ${ }^{45}$. Two important members of the family include nerve growth factor (NGF) and brain-derived neurotrophic factor (BDNF). The two proteins have similar, though not identical, mechanisms of action. NGF has three binding states: one is a tyrosine receptor kinase (Trk) homodimer, meaning that it links two Trk receptors, specifically TrkA. Another is a TrkA heterodimer with a membrane protein called p75 neurotrophin receptor (p75NTR). It can also bind independently to p75NTR. BDNF seems to have two binding states: the Trk-p75NTR heterodimer, and an independent binding to p75NTR. These two growth factors can promote cell survival through two common mechanisms. The Trk-dependent pathway involves neurotrophins binding to a Trk, either in a homodimer or heterodimer conformation. This results in phosphorylation and activation of Akt, a highly conserved pathway that results in activation and nuclear translocation of prosurvival and pro-differentiation transcription factors. The Trk-independent pathway involves the binding of p75NTR and the generation of ceramide, a sphingolipid which can be proapoptotic or anti-apoptotic depending on the context and cell type. A representative diagram of these pathways is shown in Figure 2. For a detailed review of the pathways, please see Chao et al ${ }^{45}$.

Anesthetic drugs have been shown to act on neurotrophin pathways, and extensive work has implicated the BDNF dependent pathways. Lu and coworkers examined the effect of the "triple cocktail" (midazolam, nitrous oxide, and isoflurane) in varying time lengths on BDNF expression and TrkB receptor activation in PND7 rats. Decreased expression of the downstream pro-survival effector Akt in the thalamus and cortex was found along with increased levels of caspase- 9 and caspase- 3 expression, implying activation of the intrinsic apoptotic cascade ${ }^{46}$. Interestingly, this change may be induced by different mechanisms in different brain regions. In the thalamus, decreased BDNF release led to decreased Akt expression, whereas in the cerebral cortex a large increase in ceramide production was observed, implying activation of the p75NTR Trk-independent pathway, in this case leading to apoptosis.

Head further elaborated on the role of BDNF, differentiating between proBDNF and BDNF. The precursor protein is released into the synaptic cleft, at which point it is proteolytically cleaved by tissue plasminogen activator (tPA) to the mature form mBDNF. ProBDNF activates apoptotic pathways through Trk-independent signaling, while mBDNF promotes cell survival through the Akt pathway ${ }^{47}$. They exposed five-day-old primary neurons to isoflurane and measured a dose dependent decrease in tPA secretion into the synapse. As they added tPA, the isoflurane-mediated apoptosis became markedly reduced in a dosedependent fashion. Further, using interference RNA to block expression of p75NTR resulted in a significant decrease of isoflurane-mediated apoptosis. They attributed this to a 
predominance of proBDNF in the synapse resulting in Trk-independent activation and resultant apoptosis, though they did not measure proBDNF, mBDNF, or ceramide in the studies. They further noted that tPA itself has cell signaling activity which is independent of its proteolytic activities, and also activates the Akt and ERK pathways.

While the most investigations of anesthetic actions on growth factors have involved the BDNF pathway, there is evidence that the closely related NGF pathway may also be implicated in PAN. Pesic et al. found decreased expression of NGF mRNA and protein in PND7 rats, leading to decreased phosphorylated Akt in concert with the typical apoptotic picture with propofol exposure ${ }^{37,38}$. Popic looked at PND14 rats and found variable expression and phosphorylation of the growth factors and their receptors between the thalamus and cortex but found commonality in activation of the downstream effectors Akt and ERK, which they hypothesized may have prevented the apoptosis seen in PND7 rats with a single dose of propofol. Milanovic looked at proNGF and p75NTR and found increased levels of expression after propofol exposure in PND7 rats, but also found an increased level of pAkt and another inhibitor of apoptosis, X-linked inhibitor of apoptosis protein (XIAP), which is in contrast to other studies showing decreased levels of activated Akt in response to anesthetic administration ${ }^{48}$.

Numerous studies have shown that anesthetics alter signaling downstream from the p75NTR, a receptor common to both BDNF and NGF. For example, Pearn showed that propofol induced neuroapoptosis in five day old primary murine neurons, but the effect was blocked by inhibitors of p75NTR ${ }^{49}$. Further, primary neurons from p75NTR knockout mice also did not display propofol-induced apoptosis. Furthermore, transfection of p75NTR knockout neurons with p75NTR via lentivirus restored the apoptotic response to propofol. This implies that activation of p75NTR plays a prominent role in the apoptosis induced by anesthetics. Another downstream effector of p75NTR is Rho kinase A, a protein responsible for actin depolymerization and growth cone collapse when activated. Lemkuil found evidence for RhoKA activation through a p75NTR mechanism and subsequent actin depolymerization and cell death with exposure to isoflurane ${ }^{50}$. Both of these effects were prevented by inhibition of the receptor and addition of a cytoskeletal stabilizer.

Most studies on growth factor mechanisms of PAN have focused on the effects that anesthetics may have on neuronal apoptosis, but there is emerging evidence that this mechanism may be relevant to other toxicity phenotypes. Using a neuron-glia co-culture model, Ryu and coworkers showed neurons cultured with glia that were treated with isoflurane exhibited reduced axonal growth. Supernatant from the glial cultures had reduced amounts of BDNF, and normal levels of growth were restored by adding exogenous BDNF to the co-cultures ${ }^{51}$. In an accompanying manuscript the same group has demonstrated that sevoflurane inhibited the motility of neuronal growth cones, and that motility could be restored with either a p75NTR peptide inhibitor, or further downstream in the same pathway via a Rho Kinase inhibitor (Ryu et al. 2016, this JNA). Co-immunoprecipitation experiments performed in cultured neurons exposed to sevoflurane showed enhanced binding of P75NTR to Rho-GDP dissociation inhibitor, thus suggesting a possible mechanism by which sevoflurane acts on the Rho pathway downstream from P75NTR. 
Taken together, these data convincingly demonstrate that anesthetics have profound effects on growth factors and their downstream signaling pathways, and that this in turn may result either in cell death or in a disruption of neural development. While the mechanism is plausible, nearly all of these data have been generated by experiments using in vitro systems, which may or may not be recapitulated in vivo. Further progress on growth factors as a target for anesthetic actions in PAN will require whole animal experimentation and evidence that well-established phenotypes of impaired learning and memory can be rescued via interventions in growth factor pathways.

\section{GABA Receptors}

General anesthetic agents are believed to induce unconsciousness primarily through their actions on ligand gated ion channels, and thus it is not surprising that mechanistic studies of PAN have identified these receptors as a possible target. GABA-A receptors, which contain a chloride channel, are the most abundant fast inhibitory neurotransmitter receptors in the mature central nervous system ${ }^{52}$ and possess several binding sites that interact with many of the currently used general anesthetics. Functional GABA-A receptors are expressed in neurons as early as the embryonic stage of development ${ }^{53}$. In contrast with their role in adult neurons, activation of GABA-A receptors during early development produces robust membrane depolarization in immature neurons due to an elevated level of intracellular chloride ions and efflux through chloride-permeable channels ${ }^{54}$. This is of particular concern as studies have shown that the excitatory effects of GABA-A receptor-mediated depolarization drives spontaneous synchronous activity that is fundamentally important for proper circuit formation in the developing brain ${ }^{55,56}$. Isoflurane, sevoflurane, midazolam and propofol, which are among the best studied anesthetics in PAN, act primarily by enhancing inhibitory neurotransmission via the GABA receptor ${ }^{57}$. Thus it has been hypothesized that general anesthetics may act on GABA-A receptors to disrupt normal circuits that require precise timing of GABAergic input in the developing brain.

There is evidence that GABA receptors activated by anesthetics may be implicated in initiation of apoptosis pathways in developing neurons. Using calcium-imaging techniques, Kahraman et al. found that propofol induces calcium influx that can be blocked with either bicuculline or nifedipine, which are GABA-A and L-type calcium channel antagonists, respectively. An increase in caspase-3 activity, as measured by a fluorescent probe, and in numbers of apoptotic nuclei as measured by propidium iodide/Hoechst 33258 staining was observed with propofol treatment and this effect was lessened by co-treatment with bicuculline or nifedipine ${ }^{14}$. These effects were operative only at 4 days in vitro, and were not seen in more mature neurons at day 8 . In a similar study in neuronal culture, Zhao and coworkers used calcium imaging to demonstrate that isoflurane causes GABA-dependent depolarization, which leads to calcium influx. They made a causal link to isoflurane induced activation of apoptosis pathways by showing that levels of cleaved caspase- 3 mRNA are reduced when isoflurane is co-administered with either GABA receptor or calcium channel antagonists ${ }^{58}$. While the effect sizes in these studies are not large, the concurrence between them is striking and strongly suggestive that GABA receptors may play a role in anestheticinduced neuronal apoptosis. 
Anesthetic actions at GABA receptors may also have sublethal effects that disrupt neuronal development. De Roo and coworkers have shown an abnormal increase in spine density in a rat in vivo model that occurs with developmental exposure to both isoflurane and midazolam ${ }^{59}$. While isoflurane has been shown to have many off-target effects, the actions of midazolam are generally considered to be specific to the GABA-A receptor. The same group has demonstrated similar effects using propofol, another GABAergic anesthetic ${ }^{35}$. In another study of the sublethal effects of anesthetics critical functions in brain development, Mintz et al. found that isoflurane, sevoflurane, propofol, and midazolam disrupted growth cone responses to the repulsive guidance cues Semaphorin 3A in vitro. Using an ex-vivo model of axon guidance, they showed that isoflurane completely disrupted targeted growth, and that this effect could be recapitulated with a GABA-A specific agonist, muscimol, and that targeted growth could be restored using picrotoxin, a chloride channel blocker ${ }^{60}$.

Because many anesthetic agents act on GABA receptors and GABA plays a critical role in brain development, it is highly likely that GABA receptors are an important mechanistic target in PAN. At present, the evidence for a GABA-mediated effect on neuronal apoptosis is limited to high quality in vitro studies that show strong evidence for causation. In vivo data suggesting that non-lethal anesthetic actions on GABA receptors alter spine formation remain correlational, but strong evidence from a combination of in vitro and ex-vivo preparations suggest that anesthetics disrupt axon guidance. However, none of these studies have directly linked anesthetic effects mediated via GABA receptors to learning and memory deficits seen in PAN, and no higher animal correlations exist at present.

\section{NMDA Receptors}

As described previously, the earliest work on developmental anesthetic neurotoxicity identified the NMDA receptor as a trigger for apoptosis when inhibited by ketamine. NMDA receptors mediate excitatory neurotransmission in central neurons and play an important role in synaptic plasticity and memory formation ${ }^{61-63}$. NMDA receptors are composed of two obligatory glycine-binding GluN1 subunits paired with two glutamate-binding GluN2 and/or glycine-binding GluN3 subunits ${ }^{64,65}$. The glutamate-binding GluN2 subunits (GluN2A-D) appear at specific developmental stages, bind distinct regulatory proteins, and are found at varied cellular locations ${ }^{66}$. NMDA receptors also play a role in enhancing signal and signaling processes via calcium influx ${ }^{67}$, and they act like coincidence receptors since their activation requires depolarization of the membrane potential to relieve voltage-dependent magnesium block and binding of both glutamate and glycine ${ }^{68-70}$. Anesthetic modulation of NMDAR function can occur at different levels. For example, ketamine is a non-competitive antagonist of the NMDA receptor $\mathrm{Ca} 2+$ channel pore ${ }^{71}$. In addition, at clinically relevant concentrations, ketamine interacts with the phencyclidine (PCP) binding $\operatorname{site}^{72}$, leading to significant inhibition of NMDA receptor activity ${ }^{73}$.

Ikonomidou et al. published the first animal study demonstrating that the use of NMDA receptor antagonists during the early stages of CNS development can cause neurotoxicity ${ }^{74}$. Specifically, when NMDA receptor antagonists (i.e. MK801, phencyclidine (PCP), ketamine, or carboxypiperazin-4-yl-propyl-1-phoshphonic acid (CPP)) are administered during a period of NMDA receptor hypersensitivity and synaptogenesis (brain growth spurt 
period), widespread apoptotic neurodegeneration is observed in the developing rat brain. Among the drugs tested in the Ikonomidou study, ketamine is widely used in pediatric anesthesia. Additional animal studies using ketamine further elucidated the dose and time dependency of neurotoxicity ${ }^{13,32,75-81}$. A variety of brain regions showed increased cell death following exposure to ketamine, including the frontal cortex, striatum, hippocampus, thalamus, and amygdala ${ }^{76,77,82}$. In rats, ketamine administered at postnatal day 7 has been shown to impair cognitive processes such as learning and memory at 2 months of age as indicated by the Morris water maze test ${ }^{83}$. Rhesus monkeys exposed to ketamine in infancy showed long-lasting cognitive deficits indicated by slower learning of pattern recognition ${ }^{79}$. These preclinical results indicate that NMDA receptor antagonists and specifically ketamine exposure during the vulnerable window of brain development can have neurotoxic effects that are long-lasting and lead to deficits in brain function.

Modulation of NMDA receptors can also occur intracellularly, and anesthetic effects on these actions may define a new mechanism for PAN. Kornau et al. showed that the cytoplasmic tail of NMDA receptor GluN2 subunits interact with the prominent postsynaptic density protein PSD-95 ${ }^{84}$ via a PDZ domain, which is a common motif that mediates anchoring of transmembrane proteins to the cytoskeleton. Fang et al. reported that clinically relevant concentrations of inhalational anesthetics, including halothane, isoflurane, and sevoflurane, dose-dependently and specifically inhibited the PDZ domain-mediated protein interactions between PSD-95 or PSD-93 and the NMDA receptor ${ }^{85}$. These findings revealed the PDZ domain as a new molecular target for inhalational anesthetics. Using a surface plasmon resonance-based BIAcore assay, binding of PSD-95 PDZ2 to NR2B C-terminus was shown to be disrupted by isoflurane in real time ${ }^{86}$. These results demonstrated that anesthetics can disrupt the PDZ domain-mediated protein interactions between PSD-95 and the NMDA receptor and suggest downstream effects on synaptic plasticity. Given the critical role of PSD-95 and NMDA receptors in synapse development, it has been proposed that anesthetics such as isoflurane and sevoflurane might interfere with brain development by disrupting the interactions between these proteins ${ }^{86}$.

There is strong evidence to suggest that activation of NMDA receptors by ketamine can lead to neuronal apoptosis, as this mechanism has been demonstrated in vivo in both rodents and primates. However, the importance of this finding is unclear for PAN, as ketamine is not typically used as a mainstay anesthetic for pediatric patients and the clinical literature does not specifically implicate this drug. The effects of anesthetics on the NMDA/PSD-95 interaction are intriguing and highly suggestive, but their relevance has yet to be demonstrated by in vitro or in vivo models of PAN.

\section{Mechanisms Related to Intracellular Calcium Regulation}

Intracellular calcium serves as a key component of signaling systems that control innumerable cellular processes. The intracellular levels of calcium are tightly controlled in local microdomains, and thus anesthetic agents might exert toxic effects on multiple aspects of neural development via their effects on calcium regulation. It is well known that calcium is a critical mediator in neuronal apoptosis ${ }^{87}$, which has been strongly implicated in PAN as discussed above. Additionally, calcium regulation is critical to nearly every important 
process in neural development, including motility of neurites and formation of synapses, both of which are known to be susceptible to anesthetic effects ${ }^{88}$.

The most notable study on calcium as a target in PAN was conducted by Wei and coworkers, who used both genetic and pharmacologic strategies to inhibit inositol 1,4,5-trisphosphate receptors (InsP3Rs) in primary cultured neurons to inhibit excess calcium release caused by isoflurane ${ }^{89}$. They found evidence of a reduction in isoflurane-induced neurotoxicity as measured by LDH and MTT assays, although they did not measure cell death directly and these changes required a high dose and long duration exposure of 24 hours at $2.4 \%$ isoflurane to be manifested. In the same report, the authors showed that pharmacologic inhibition of InsP3Rs delivered acutely prior to a 6 hour exposure to $1.0 \%$ or $1.5 \%$ isoflurane in early postnatal mice reduced caspase-3 expression and partially rescued behavioral deficits in the Morris water maze ${ }^{89}$. Taken together, these findings are strong evidence that calcium dysregulation may play a substantial role in developmental anesthetic neurotoxicity. The same group demonstrated a likely relevance to human biology using an immortalized human neural progenitor cell line. Isoflurane induced neurotoxicity could be substantially attenuated by inhibition of the release of internal calcium stores by pharmacologic inhibition of either InsP3R or the ryanodine receptor, although exposures with at least $1.2 \%$ for 24 hours or $2.4 \%$ for 12 hours were required to cause measurable toxicity in this model ${ }^{90}$. Interestingly, isoflurane preconditioning prevented calcium-induced toxicity in this model.

The evidence for potential calcium-mediated mechanisms of neurotoxicity is not limited to isoflurane. Ketamine blocks calcium currents by its antagonistic action at the NMDA receptor, and thus toxic effects of this agent have been proposed to be related to calcium neurotoxicity. A study of primary cultured neurons showed that a 24-hour exposure to ketamine resulted in an increase in intracellular calcium concentrations and a decrease in the frequency and amplitude of calcium oscillations, which continued for 24 hours after withdrawal of the drug. These effects correlated with a decrease in immunolabeling for synapsin, a pre-synaptic marker ${ }^{91}$. The same group found that exposure to midazolam in an identical paradigm inhibited calcium oscillations and also reduced synapsin immunolabeling ${ }^{92}$. While these studies are of interest, it is notable that they require long exposures and do not test for any effects beyond 24 hours. Furthermore, the relevance to brain development is more difficult to determine as the authors used neuronal cultures at 15 days in vitro, at which time many developmental phenomenon in primary neuron cultures, such as synaptogenesis, have already reached completion.

Basic anesthetic pharmacology and an appreciation of the far-reaching effects of calcium in signaling systems relevant to brain development both suggest that calcium is likely to play a role in PAN. At this time, studies are limited to the rodent model, and further exploration in this system is warranted prior to progression to more complex systems. It is notable that both principal lines of investigation related to this mechanism use very long durations of exposure, and a key question is whether this mechanism is operative in the time frames more commonly associated with anesthesia exposure in the operating room, or whether it might have greater relevance to toxicity induced by sedation in an intensive care setting rather than PAN. 


\section{Effects on Mitochondria}

Newly emerging data have focused attention on whether anesthetics might disrupt brain development via actions on mitochondrial function. Mitochondria are double-membrane bound organelles common to eukaryotic cells, which produce adenosine triphosphate (ATP) to power cellular activities via the electron transport chain located in the inner membrane. Additionally, mitochondria are involved in numerous signaling systems, cellular growth, differentiation, and cell cycle regulation ${ }^{93}$. Mitochondrial defects have been implicated in autism spectrum disorders (ASDs) ${ }^{94}$, thus showing that a disruption of this organelle can result in neurodevelopmental defects. It has been appreciated for some time that anesthetics act on mitochrondria ${ }^{95}$, and thus investigators have asked whether mitochondria might be involved in the mechanism of pediatric anesthetic neurotoxicity.

In a 2010 study by Lunardi and co-workers ${ }^{96}$ to assess the chronic effects of anesthetic exposure on synapses, electron microscopy revealed evidence of mitochrondrial degeneration in neurons of the hippocampal subiculum. In this study, postnatal day 7 (PND 7) rat pups underwent 6 hours of anesthesia with isoflurane, nitrous oxide, and midazolam, and experiments were conducted at postnatal day 21 (PND 21). The authors reported seeing an increase in mitochondria which were either swollen with normal membranes or shrunken with a loss of resolution of the inner and outer membranes, but did not present quantitative data to represent their findings. In a follow-up study using the same paradigm of exposure, a quantitative analysis of the morphology and density of mitochondria was conducted, which found enlarged mitochondria and decreased mitochondrial concentration at presynaptic terminals ${ }^{97}$. Using spectrophotometry, the authors also showed a significant reduction in the activity of Complex IV, a critical component of the electron transport chain. They went on to show changes in synaptic transmission via electrophysiology in acute brain slices. While it is logical that the observed decrease in inhibitory synaptic neurotransmission may be due to the observed effects on the mitochondria, other explanations are not excluded by these data. Interestingly, in a recent paper, $\mathrm{Xu}$ showed that sevoflurane-induced apoptosis in primary cultured neurons could be prevented using a mitochondrial division inhibitor ${ }^{98}$. At this point, there is strong evidence to suggest that early anesthetic exposure strongly affects mitochondrial structure and function in neurons, and there is emerging evidence that mitochondrial disturbance may be associated with decreased synaptic transmission, a known inducer of apoptosis in developing neurons.

While data on mitochondrial mechanisms of PAN is not extensive, the extant work is of high quality in terms of study design and in the degree to which published findings are consistent with each other. This subfield deserves further study in the rodent, which should be rapidly extended to higher animal models if it continues to be promising.

\section{Epigenetic Mechanisms}

Brain development requires highly regulated control of gene expression in a timing-, region-, and cell-specific manner in order to coordinate the myriad processes required to produce a fully functioning central nervous system. These gene expression programs are orchestrated in large part by epigenetic mechanisms, and thus recent reports that anesthetics can alter 
these processes ${ }^{99}$ suggest a potent, little-explored mechanism by which they might interfere with brain development. The term "epigenetics" refers to alterations in DNA structure that affect gene expression without changing the DNA sequence itself. More simplistically, epigenetics can be thought of as molecular "switches" that turn genes on and off. The two primary forms of epigenetic modifications are DNA methylation and histone modifications. DNA methylation located within gene promoter regions is associated with decreases in transcription ${ }^{100}$. Histone modifications occur when the amino-terminal tails of histone proteins are subject to an array of posttranslational modifications, including acetylation, methylation, phosphorylation, ubiquination, and SUMOlation, and these modifications individually or in concert - recruit effector proteins, through which they exert their effects on transcription ${ }^{101}$.

Emerging evidence suggests that anesthetic agents are capable of inducing epigenetic changes in brain during development and that these changes might be responsible for cognitive deficits. A recent study showed that exposure of mice to isoflurane on postnatal days 7 through 9 resulted in memory deficits 3 months later that correlated with dysregulated histone acetylation in hippocampus ${ }^{102}$. Though an interesting and important finding, this study examined global histone acetylation employing Western blot, which does not provide information regarding the genomic location of these histone changes. To date, only one study has examined epigenetic changes at specific gene loci. Massara found that treatment of P7 rats with midazolam, nitrous oxide, and isoflurane for 6 hours resulted in a global decrease in acetylated $\mathrm{H} 3 \mathrm{~K} 4$ in hippocampus ${ }^{103}$. They also found that $\mathrm{H} 3 \mathrm{~K} 4$ acetylation was specifically decreased within the promoter regions of c-Fos and Bdnf - two genes implicated in brain development - and this was associated with a parallel decline in transcripts. Furthermore, treatment of cultured hippocampal neurons with a histone deacetylase inhibitor (HDACi) reversed GA-induced changes in cell morphology, while treatment of hippocampal slice cultures with HDACi reversed GA-induced impairment of postsynaptic GABAA receptor-mediated currents. Another recent study by Wu et al. also showed that isoflurane exposure in postnatal day 7 rats results in changes in histone deacetylase 2, methyl-cytosine-phosphate-guanine-binding protein 2, and DNA methyltransferase interactions resulting in reduced expression of BDNF in the hippocampus. These deficits correlate with impairments in synaptic plasticity and reduced performance in behavioral assays of learning, and they can be reversed by caging in an enriched environment ${ }^{104}$.

While the above mentioned studies provide exciting new evidence that exposure to anesthetics during critical neurodevelopmental windows alters epigenetic processes in the hippocampus, which subsequently impacts gene transcription and higher cognitive processes, a great deal of work still remains to be done. The histone code is incredibly complex, comprised of over 100 different modifications. With the advent of next generation sequencing, it has become possible to generate genome-wide histone modification maps employing chromatin immunoprecipitation followed by deep sequencing ("ChIP-Seq) $)^{105,106}$ [Park 2009; Barski 2007], and this approach should be employed to gain a more complete picture. Furthermore, translation of these findings to higher animals is necessary, but will be complicated by the absence of model systems in non-human primates and other animal models with complex gyrated brains. 


\section{Inflammatory Mechanisms}

Recent work in the neurosciences has revealed that neuronal development may be profoundly impacted by inflammation in ways that are relevant both to normal development and disease ${ }^{107}$, and thus inflammation is an area of interest in studies of PAN. The central nervous system (CNS) was once thought to be a site of absolute immunologic privilege, i.e. immunologic effector cells are unable to cross the blood-brain barrier (BBB) and access the CNS. However, it is now known that immunologic privilege is relative and that active immune surveillance occurs within the $\mathrm{CNS}^{108}$. To access the CNS, immune cells (e.g., leukocytes) must penetrate the endothelial BBB, epithelial blood-cerebrospinal fluid (CSF) barrier, or the tanycytic barrier (surrounding circumventricular organs). Microglia, the resident innate immune cells of the CNS, are exquisite sensors of their environment. Upon recognition of pathogens or local tissue damage, microglia activate within hours of the injury and accumulate at the site of damage. Once activated, they become competent antigen presenting cells and effector cells within the environment, performing a range of actions. They may promote neuronal survival via release of trophic factors, removal of toxic cellular debris, and guided migration of neural stem cells to the site of injury. Alternatively, activated microglia may promote neuronal damage by their release of reactive oxygen species and pro-inflammatory cytokines ${ }^{109}$.

General anesthetic agents may have pro-inflammatory and anti-inflammatory effects depending on the drug, dose, and target tissue. Isoflurane has anti-inflammatory effects in all contexts that have been studied except neuronal cell lines ${ }^{110,111}$. Outside the central nervous system, isoflurane and sevoflurane display anti-inflammatory effects in adult rodent models of sepsis, cerebral ischemia, and cardiac ischemia ${ }^{111}$. Within the CNS, isoflurane activates a neuroinflammatory response in adult rodent neurons, characterized by increased production of tumor necrosis factor alpha (TNFa), interleukin 1 beta (IL-1 $\beta$ ), and interleukin 6 (IL-6) in brain and serum ${ }^{12,113}$. The increased release of TNFa in response to volatile anesthetics was even more pronounced in a mouse model of Alzheimer's disease ${ }^{113}$, suggesting that animals predisposed to cognitive impairment were more susceptible. Isoflurane and sevoflurane increased levels of IL-6, nuclear levels of the pro-inflammatory transcription factor nuclear factor kappa beta (NF-KB), and transcription activity of NF-KB H4 human neuroglioma cells and microglia. NF-KB inhibitors attenuated volatile anesthetic-induced increases in IL-6 and NF-KB, suggesting a role for NF-KB signaling within volatile anesthetic-induced increases of inflammatory cytokines ${ }^{114}$.

While these data suggest a role for inflammation in anesthetic-induced neurotoxicity in mature animals, their significance in the developmental context is unknown. Extensive further work in pre-clinical models is required in order to determine how inflammation might be relevant to PAN, and this hypothesis remains one of the least developed, but one of the most promising.

\section{Conclusion}

Although considerable effort has been directed towards animal model research, no consensus yet exists as to the mechanism of pediatric anesthetic neurotoxicity. This is 
perhaps inevitable as general anesthetic drugs typically have many off-target effects, and different investigators have chosen to focus on a range of phenotypes, including cell death, alterations in synapse number or function, and changes in neurite development. It is conceivable that multiple distinct mechanisms contribute to the deficits in spatial learning that are common within the animal literature, but it is more likely that some potential mechanisms are epiphenomena. Ideally at the conclusion of a review article such as this one, it would be possible to draw a diagram conclusively relating the different proposed mechanisms, but at present we can only note common themes. It is striking that BDNF has been implicated in a wide variety of mechanistic studies, and it seems likely that anesthetic actions affecting this critical trophic factor will prove important. Numerous studies point to a variety of mechanisms by which anesthetics activate pro-apoptotic pathways, but it remains unclear whether apoptosis is a cause or consequence of PAN. In fact, it is probably not plausible to define a mechanism of PAN until the phenotype of the injury has become clear, and at present numerous competing hypotheses exist ${ }^{115}$. Future work on mechanisms of toxicity should be directed towards settling this question, extending currently identified putative mechanisms towards more advanced model systems, and to asking how existing mechanisms could be inter-related.

\section{References}

1. Rappaport B, Mellon RD, Simone A, Woodcock J. Defining safe use of anesthesia in children. The New England journal of medicine. 2011; 364(15):1387-1390. [PubMed: 21388302]

2. Kuehn, BM. Jama. Vol. 305. United States: 2011. FDA considers data on potential risks of anesthesia use in infants, children; p. 1749-1750.1753

3. Ramsay, JG.; Rappaport, BA. Anesth Analg. Vol. 113. United States: 2011. SmartTots: a multidisciplinary effort to determine anesthetic safety in young children; p. 963-964.

4. Wilder RT, Flick RP, Sprung J, et al. Early exposure to anesthesia and learning disabilities in a population-based birth cohort. Anesthesiology. 2009; 110(4):796-804. [PubMed: 19293700]

5. Flick RP, Katusic SK, Colligan RC, et al. Cognitive and behavioral outcomes after early exposure to anesthesia and surgery. Pediatrics. 2011; 128(5):e1053-1061. [PubMed: 21969289]

6. DiMaggio C, Sun LS, Kakavouli A, Byrne MW, Li G. A retrospective cohort study of the association of anesthesia and hernia repair surgery with behavioral and developmental disorders in young children. Journal of neurosurgical anesthesiology. 2009; 21(4):286-291. [PubMed: 19955889]

7. DiMaggio C, Sun LS, Li G. Early childhood exposure to anesthesia and risk of developmental and behavioral disorders in a sibling birth cohort. Anesth Analg. 2011; 113(5):1143-1151. [PubMed: 21415431]

8. Ing C, DiMaggio C, Whitehouse A, et al. Long-term differences in language and cognitive function after childhood exposure to anesthesia. Pediatrics. 2012; 130(3):e476-485. [PubMed: 22908104]

9. Ikonomidou C, Bosch F, Miksa M, et al. Blockade of NMDA Receptors and Apoptotic Neurodegeneration in the Developing Brain. Science. 1999; 283(5398):70-74. [PubMed: 9872743]

10. Jevtovic-Todorovic, V.; Hartman, RE.; Izumi, Y., et al. J Neurosci. Vol. 23. United States: 2003. Early exposure to common anesthetic agents causes widespread neurodegeneration in the developing rat brain and persistent learning deficits; p. 876-882.

11. Yon JH, Daniel-Johnson J, Carter LB, Jevtovic-Todorovic V. Anesthesia induces neuronal cell death in the developing rat brain via the intrinsic and extrinsic apoptotic pathways. Neuroscience. 2005; 135(3):815-827. [PubMed: 16154281]

12. Ma D, Williamson P, Januszewski A, et al. Xenon mitigates isoflurane-induced neuronal apoptosis in the developing rodent brain. Anesthesiology. 2007; 106(4):746-753. [PubMed: 17413912] 
13. Slikker W Jr. Zou X, Hotchkiss CE, et al. Ketamine-induced neuronal cell death in the perinatal rhesus monkey. Toxicological sciences : an official journal of the Society of Toxicology. 2007; 98(1):145-158. [PubMed: 17426105]

14. Kahraman S, Zup SL, McCarthy MM, Fiskum G. GABAergic Mechanism of Propofol Toxicity in Immature Neurons. Journal of neurosurgical anesthesiology. 2008; 20(4):233-240. [PubMed: 18812886]

15. Brambrink AM, Evers AS, Avidan MS, et al. Ketamine-induced neuroapoptosis in the fetal and neonatal rhesus macaque brain. Anesthesiology. 2012; 116(2):372-384. [PubMed: 22222480]

16. Brambrink AM, Evers AS, Avidan MS, et al. Isoflurane-induced Neuroapoptosis in the Developing Neonatal Rhesus Macaque Brain. Anesthesiology. 2010; 112(4):834-841. [PubMed: 20234312]

17. Raper J, Alvarado MC, Murphy KL, Baxter MG. Multiple Anesthetic Exposure in Infant Monkeys Alters Emotional Reactivity to an Acute Stressor. Anesthesiology. 2015; 123(5):1084-1092. [PubMed: 26313293]

18. Zhou Z, Ma D. Anaesthetics-Induced Neurotoxicity in Developing Brain: An Update on Preclinical Evidence. Brain Sciences. 2014; 4(1):136-149. [PubMed: 24961704]

19. Lossi L, Merighi A. In vivo cellular and molecular mechanisms of neuronal apoptosis in the mammalian CNS. Progress in Neurobiology. 2003; 69(5):287-312. [PubMed: 12787572]

20. Nijhawan D, Honarpour N, Wang X. Apoptosis in neural development and disease. Annual review of neuroscience. 2000; 23:73-87.

21. Banasiak KJ, Xia Y, Haddad GG. Mechanisms underlying hypoxia-induced neuronal apoptosis. Prog Neurobiol. 2000; 62(3):215-249. [PubMed: 10840148]

22. Valencia A, Moran J. Reactive oxygen species induce different cell death mechanisms in cultured neurons. Free radical biology \& medicine. 2004; 36(9):1112-1125. [PubMed: 15082065]

23. Nicotera P, Orrenius S. The role of calcium in apoptosis. Cell calcium. 1998; 23(2-3):173-180. [PubMed: 9601613]

24. Allsopp TE, Scallan MF, Williams A, Fazakerley JK. Virus infection induces neuronal apoptosis: A comparison with trophic factor withdrawal. Cell death and differentiation. 1998; 5(1):50-59. [PubMed: 10200445]

25. Gobbel GT, Bellinzona M, Vogt AR, Gupta N, Fike JR, Chan PH. Response of postmitotic neurons to X-irradiation: implications for the role of DNA damage in neuronal apoptosis. The Journal of neuroscience : the official journal of the Society for Neuroscience. 1998; 18(1):147-155. [PubMed: 9412495]

26. Tesh VL. Induction of apoptosis by Shiga toxins. Future microbiology. 2010; 5:431-453. [PubMed: 20210553]

27. Czabotar PE, Lessene G, Strasser A, Adams JM. Control of apoptosis by the BCL-2 protein family: implications for physiology and therapy. Nature reviews. Molecular cell biology. 2014; 15(1):49-63. [PubMed: 24355989]

28. Varfolomeev EE, Ashkenazi A. Tumor Necrosis Factor: An Apoptosis JuNKie? Cell. 2004; 116(4): 491-497. [PubMed: 14980217]

29. Martin DC, Plagenhoef M, Abraham J, Dennison RL, Aronstam RS. Volatile anesthetics and glutamate activation of $N$-methyl-D-aspartate receptors. Biochemical Pharmacology. 1995; 49(6): 809-817. [PubMed: 7702639]

30. Ogata J, Shiraishi M, Namba T, Smothers CT, Woodward JJ, Harris RA. Effects of anesthetics on mutant N-methyl-D-aspartate receptors expressed in Xenopus oocytes. The Journal of pharmacology and experimental therapeutics. 2006; 318(1):434-443. [PubMed: 16622040]

31. Ikonomidou C, Bittigau P, Ishimaru MJ, et al. Ethanol-induced apoptotic neurodegeneration and fetal alcohol syndrome. Science. 2000; 287(5455):1056-1060. [PubMed: 10669420]

32. Young C, Jevtovic-Todorovic V, Qin YQ, et al. Potential of ketamine and midazolam, individually or in combination, to induce apoptotic neurodegeneration in the infant mouse brain. British journal of pharmacology. 2005; 146(2):189-197. [PubMed: 15997239]

33. Rizzi S, Carter LB, Ori C, Jevtovic-Todorovic V. Clinical anesthesia causes permanent damage to the fetal guinea pig brain. Brain pathology (Zurich, Switzerland). 2008; 18(2):198-210.

34. Bercker S, Bert B, Bittigau P, et al. Neurodegeneration in newborn rats following propofol and sevoflurane anesthesia. Neurotoxicity research. 2009; 16(2):140-147. [PubMed: 19526290] 
35. Briner A, De Roo M, Dayer A, Muller D, Habre W, Vutskits L. Volatile anesthetics rapidly increase dendritic spine density in the rat medial prefrontal cortex during synaptogenesis. Anesthesiology. 2010; 112(3):546-556. [PubMed: 20124985]

36. Cattano D, Young C, Straiko MM, Olney JW. Subanesthetic doses of propofol induce neuroapoptosis in the infant mouse brain. Anesth Analg. 2008; 106(6):1712-1714. [PubMed: 18499599]

37. Pesic V, Milanovic D, Tanic N, et al. Potential mechanism of cell death in the developing rat brain induced by propofol anesthesia. International journal of developmental neuroscience : the official journal of the International Society for Developmental Neuroscience. 2009; 27(3):279-287. [PubMed: 19150648]

38. Popic J, Pesic V, Milanovic D, et al. Propofol-induced changes in neurotrophic signaling in the developing nervous system in vivo. PLoS One. 2012; 7(4):e34396. [PubMed: 22496799]

39. Yu D, Jiang Y, Gao J, Liu B, Chen P. Repeated exposure to propofol potentiates neuroapoptosis and long-term behavioral deficits in neonatal rats. Neuroscience letters. 2013; 534:41-46. [PubMed: 23295901]

40. Zhang G, Dong Y, Zhang B, et al. Isoflurane-induced caspase-3 activation is dependent on cytosolic calcium and can be attenuated by memantine. The Journal of neuroscience : the official journal of the Society for Neuroscience. 2008; 28(17):4551-4560. [PubMed: 18434534]

41. Akerman CJ, Cline HT. Refining the roles of GABAergic signaling during neural circuit formation. Trends in neurosciences. 2007; 30(8):382-389. [PubMed: 17590449]

42. Nyman Y, Fredriksson A, Lonnqvist PA, Viberg H. Etomidate exposure in early infant mice (P10) does not induce apoptosis or affect behaviour. Acta Anaesthesiol Scand. 2016; 60(5):588-596. [PubMed: 26763687]

43. Lu Y, Huang Y, Jiang J, et al. Neuronal apoptosis may not contribute to the long-term cognitive dysfunction induced by a brief exposure to $2 \%$ sevoflurane in developing rats. Biomedicine \& Pharmacotherapy. 2016; 78:322-328. [PubMed: 26898457]

44. Kilkenny C, Browne WJ, Cuthill IC, Emerson M, Altman DG. Improving Bioscience Research Reporting: The ARRIVE Guidelines for Reporting Animal Research. PLoS Biol. 2010; 8(6):e1000412. [PubMed: 20613859]

45. Chao MV. Neurotrophins and their receptors: a convergence point for many signalling pathways. Nat Rev Neurosci. 2003; 4(4):299-309. [PubMed: 12671646]

46. Lu LX, Yon JH, Carter LB, Jevtovic-Todorovic V. General anesthesia activates BDNF-dependent neuroapoptosis in the developing rat brain. Apoptosis : an international journal on programmed cell death. 2006; 11(9):1603-1615. [PubMed: 16738805]

47. Je HS, Yang F, Ji Y, Nagappan G, Hempstead BL, Lu B. Role of pro-brain-derived neurotrophic factor (proBDNF) to mature BDNF conversion in activity-dependent competition at developing neuromuscular synapses. Proceedings of the National Academy of Sciences. 2012; 109(39): 15924-15929.

48. Milanovic D, Pesic V, Popic J, et al. Propofol anesthesia induces proapoptotic tumor necrosis factor-alpha and pro-nerve growth factor signaling and prosurvival Akt and XIAP expression in neonatal rat brain. J Neurosci Res. 2014; 92(10):1362-1373. [PubMed: 24827783]

49. Pearn ML, Hu Y, Niesman IR, et al. Propofol neurotoxicity is mediated by p75 neurotrophin receptor activation. Anesthesiology. 2012; 116(2):352-361. [PubMed: 22198221]

50. Lemkuil BP, Head BP, Pearn ML, Patel HH, Drummond JC, Patel PM. Isoflurane Neurotoxicity Is Mediated by p75(NTR)-RhoA Activation and Actin Depolymerization. Anesthesiology. 2011; 114(1):49-57. [PubMed: 21169791]

51. Ryu YK, Khan S, Smith SC, Mintz CD. Isoflurane impairs the capacity of astrocytes to support neuronal development in a mouse dissociated coculture model. Journal of neurosurgical anesthesiology. 2014; 26(4):363-368. [PubMed: 25191957]

52. Garcia PS, Kolesky SE, Jenkins A. General anesthetic actions on GABA(A) receptors. Current neuropharmacology. 2010; 8(1):2-9. [PubMed: 20808541]

53. Laurie DJ, Wisden W, Seeburg PH. The distribution of thirteen GABAA receptor subunit mRNAs in the rat brain. III. Embryonic and postnatal development. The Journal of neuroscience : the official journal of the Society for Neuroscience. 1992; 12(11):4151-4172. [PubMed: 1331359] 
54. Ben-Ari Y. Excitatory actions of gaba during development: the nature of the nurture. Nat Rev Neurosci. 2002; 3(9):728-739. [PubMed: 12209121]

55. Leinekugel X, Khazipov R, Cannon R, Hirase H, Ben-Ari Y, Buzsaki G. Correlated bursts of activity in the neonatal hippocampus in vivo. Science. 2002; 296(5575):2049-2052. [PubMed: 12065842]

56. Cherubini E, Griguoli M, Safiulina V, Lagostena L. The depolarizing action of GABA controls early network activity in the developing hippocampus. Mol Neurobiol. 2011; 43(2):97-106. [PubMed: 21042953]

57. Krasowski MD, Harrison NL. General anaesthetic actions on ligand-gated ion channels. Cellular and molecular life sciences : CMLS. 1999; 55(10):1278-1303. [PubMed: 10487207]

58. Zhao YL, Xiang Q, Shi QY, et al. GABAergic excitotoxicity injury of the immature hippocampal pyramidal neurons' exposure to isoflurane. Anesth Analg. 2011; 113(5):1152-1160. [PubMed: 21918167]

59. De Roo M, Klauser P, Briner A, et al. Anesthetics rapidly promote synaptogenesis during a critical period of brain development. PLoS One. 2009; 4(9):e7043. [PubMed: 19756154]

60. Mintz CD, Barrett KM, Smith SC, Benson DL, Harrison NL. Anesthetics interfere with axon guidance in developing mouse neocortical neurons in vitro via a gamma-aminobutyric acid type A receptor mechanism. Anesthesiology. 2013; 118(4):825-833. [PubMed: 23364597]

61. Collingridge GL, Singer W. Excitatory amino acid receptors and synaptic plasticity. Trends in pharmacological sciences. 1990; 11(7):290-296. [PubMed: 2167544]

62. Bourne HR, Nicoll R. Molecular machines integrate coincident synaptic signals. Cell. 1993; 72(Suppl):65-75. [PubMed: 8094038]

63. Kennedy MB. The biochemistry of synaptic regulation in the central nervous system. Annual review of biochemistry. 1994; 63:571-600.

64. Paoletti P, Bellone C, Zhou Q. NMDA receptor subunit diversity: impact on receptor properties, synaptic plasticity and disease. Nat Rev Neurosci. 2013; 14(6):383-400. [PubMed: 23686171]

65. Traynelis SF, Wollmuth LP, McBain CJ, et al. Glutamate receptor ion channels: structure, regulation, and function. Pharmacol Rev. 2010; 62(3):405-496. [PubMed: 20716669]

66. Hardingham GE, Bading H. Synaptic versus extrasynaptic NMDA receptor signalling: implications for neurodegenerative disorders. Nat Rev Neurosci. 2010; 11(10):682-696. [PubMed: 20842175]

67. Cotman CW, Monaghan DT, Ganong AH. Excitatory amino acid neurotransmission: NMDA receptors and Hebb-type synaptic plasticity. Annual review of neuroscience. 1988; 11:61-80.

68. Mayer ML, Westbrook GL, Guthrie PB. Voltage-dependent block by Mg2+ of NMDA responses in spinal cord neurones. Nature. 1984; 309(5965):261-263. [PubMed: 6325946]

69. Nowak L, Bregestovski P, Ascher P, Herbet A, Prochiantz A. Magnesium gates glutamate-activated channels in mouse central neurones. Nature. 1984; 307(5950):462-465. [PubMed: 6320006]

70. Johnson JW, Ascher P. Glycine potentiates the NMDA response in cultured mouse brain neurons. Nature. 1987; 325(6104):529-531. [PubMed: 2433595]

71. Hall R, Murdoch J. Brain protection: physiological and pharmacological considerations. Part II: The pharmacology of brain protection. Canadian journal of anaesthesia = Journal canadien d'anesthesie. 1990; 37(7):762-777.

72. Smith DJ, Bouchal RL, deSanctis CA, et al. Properties of the interaction between ketamine and opiate binding sites in vivo and in vitro. Neuropharmacology. 1987; 26(9):1253-1260. [PubMed: 2823161]

73. Brockmeyer DM, Kendig JJ. Selective effects of ketamine on amino acid-mediated pathways in neonatal rat spinal cord. British journal of anaesthesia. 1995; 74(1):79-84. [PubMed: 7880712]

74. Ikonomidou C, Bosch F, Miksa M, et al. Blockade of NMDA receptors and apoptotic neurodegeneration in the developing brain. Science. 1999; 283(5398):70-74. [PubMed: 9872743]

75. Hayashi H, Dikkes P, Soriano SG. Repeated administration of ketamine may lead to neuronal degeneration in the developing rat brain. Paediatric anaesthesia. 2002; 12(9):770-774. [PubMed: 12519135]

76. Zou X, Patterson TA, Sadovova N, et al. Potential neurotoxicity of ketamine in the developing rat brain. Toxicol Sci. 2009; 108(1):149-158. [PubMed: 19126600] 
77. Soriano SG, Liu Q, Li J, et al. Ketamine activates cell cycle signaling and apoptosis in the neonatal rat brain. Anesthesiology. 2010; 112(5):1155-1163. [PubMed: 20418696]

78. Liu F, Zou X, Sadovova N, et al. Changes in gene expression after phencyclidine administration in developing rats: a potential animal model for schizophrenia. Int J Dev Neurosci. 2011; 29(3):351358. [PubMed: 20691775]

79. Paule MG, Li M, Allen RR, et al. Ketamine anesthesia during the first week of life can cause longlasting cognitive deficits in rhesus monkeys. Neurotoxicology and teratology. 2011; 33(2):220230. [PubMed: 21241795]

80. Ullah N, Ullah I, Lee HY, et al. Protective function of nicotinamide against ketamine-induced apoptotic neurodegeneration in the infant rat brain. J Mol Neurosci. 2012; 47(1):67-75. [PubMed: 22160932]

81. Liu F, Patterson TA, Sadovova N, et al. Ketamine-induced neuronal damage and altered N-methylD-aspartate receptor function in rat primary forebrain culture. Toxicol Sci. 2013; 131(2):548-557. [PubMed: 23065140]

82. Zou X, Patterson TA, Divine RL, et al. Prolonged exposure to ketamine increases neurodegeneration in the developing monkey brain. International journal of developmental neuroscience : the official journal of the International Society for Developmental Neuroscience. 2009; 27(7):727-731. [PubMed: 19580862]

83. Huang L, Liu Y, Jin W, Ji X, Dong Z. Ketamine potentiates hippocampal neurodegeneration and persistent learning and memory impairment through the PKCgamma-ERK signaling pathway in the developing brain. Brain Res. 2012; 1476:164-171. [PubMed: 22985497]

84. Kornau HC, Schenker LT, Kennedy MB, Seeburg PH. Domain interaction between NMDA receptor subunits and the postsynaptic density protein PSD-95. Science. 1995; 269(5231):17371740. [PubMed: 7569905]

85. Fang M, Tao YX, He F, et al. Synaptic PDZ domain-mediated protein interactions are disrupted by inhalational anesthetics. The Journal of biological chemistry. 2003; 278(38):36669-36675. [PubMed: 12853458]

86. Tao F, Chen Q, Sato Y, Skinner J, Tang P, Johns RA. Inhalational anesthetics disrupt postsynaptic density protein-95, Drosophila disc large tumor suppressor, and zonula occludens-1 domain protein interactions critical to action of several excitatory receptor channels related to anesthesia. Anesthesiology. 2015; 122(4):776-786. [PubMed: 25654436]

87. Orrenius S, Zhivotovsky B, Nicotera P. Regulation of cell death: the calcium-apoptosis link. Nature reviews. Molecular cell biology. 2003; 4(7):552-565. [PubMed: 12838338]

88. Rosenberg SS, Spitzer NC. Calcium signaling in neuronal development. Cold Spring Harbor perspectives in biology. 2011; 3(10):a004259. [PubMed: 21730044]

89. Zhao Y, Liang G, Chen Q, et al. Anesthetic-induced neurodegeneration mediated via inositol 1,4,5trisphosphate receptors. The Journal of pharmacology and experimental therapeutics. 2010; 333(1):14-22. [PubMed: 20086058]

90. Zhao X, Yang Z, Liang G, et al. Dual effects of isoflurane on proliferation, differentiation, and survival in human neuroprogenitor cells. Anesthesiology. 2013; 118(3):537-549. [PubMed: 23314167]

91. Sinner B, Friedrich O, Zink W, Zausig Y, Graf BM. The toxic effects of s(+)-ketamine on differentiating neurons in vitro as a consequence of suppressed neuronal $\mathrm{Ca} 2+$ oscillations. Anesth Analg. 2011; 113(5):1161-1169. [PubMed: 21788311]

92. Sinner B, Friedrich O, Zausig Y, Bein T, Graf BM. Toxic effects of midazolam on differentiating neurons in vitro as a consequence of suppressed neuronal Ca2+-oscillations. Toxicology. 2011; 290(1):96-101. [PubMed: 21920406]

93. McBride HM, Neuspiel M, Wasiak S. Mitochondria: more than just a powerhouse. Current biology : CB. 2006; 16(14):R551-560. [PubMed: 16860735]

94. Legido A, Jethva R, Goldenthal MJ. Mitochondrial dysfunction in autism. Seminars in pediatric neurology. 2013; 20(3):163-175. [PubMed: 24331358]

95. Cohen PJ. Effect of anesthetics on mitochondrial function. Anesthesiology. 1973; 39(2):153-164. [PubMed: 4146381] 
96. Lunardi N, Ori C, Erisir A, Jevtovic-Todorovic V. General anesthesia causes long-lasting disturbances in the ultrastructural properties of developing synapses in young rats. Neurotoxicity research. 2010; 17(2):179-188. [PubMed: 19626389]

97. Sanchez V, Feinstein SD, Lunardi N, et al. General Anesthesia Causes Long-term Impairment of Mitochondrial Morphogenesis and Synaptic Transmission in Developing Rat Brain.

Anesthesiology. 2011; 115(5):992-1002. [PubMed: 21909020]

98. Xu F, Armstrong R, Urrego D, et al. The mitochondrial division inhibitor Mdivi-1 rescues mammalian neurons from anesthetic-induced cytotoxicity. Molecular brain. 2016; 9:35. [PubMed: 27009068]

99. Ji M, Dong L, Jia M, et al. Epigenetic enhancement of brain-derived neurotrophic factor signaling pathway improves cognitive impairments induced by isoflurane exposure in aged rats. Mol Neurobiol. 2014; 50(3):937-944. [PubMed: 24553857]

100. Schubeler D. Function and information content of DNA methylation. Nature. 2015; 517(7534): 321-326. [PubMed: 25592537]

101. Kouzarides T. SnapShot: Histone-modifying enzymes. Cell. 2007; 131(4):822. [PubMed: 18022374]

102. Zhong T, Guo Q, Zou W, et al. Neonatal isoflurane exposure induces neurocognitive impairment and abnormal hippocampal histone acetylation in mice. PLoS One. 2015; 10(4):e0125815. [PubMed: 25928815]

103. Dalla Massara L, Osuru HP, Oklopcic A, et al. General Anesthesia Causes Epigenetic Histone Modulation of c-Fos and Brain-derived Neurotrophic Factor, Target Genes Important for Neuronal Development in the Immature Rat Hippocampus. Anesthesiology. 2016

104. Wu J, Bie B, Naguib M. Epigenetic Manipulation of Brain-derived Neurotrophic Factor Improves Memory Deficiency Induced by Neonatal Anesthesia in Rats. Anesthesiology. 2016; 124(3):624640. [PubMed: 26649423]

105. Park PJ. ChIP-seq: advantages and challenges of a maturing technology. Nature reviews. Genetics. 2009; 10(10):669-680.

106. Barski A, Cuddapah S, Cui K, et al. High-resolution profiling of histone methylations in the human genome. Cell. 2007; 129(4):823-837. [PubMed: 17512414]

107. Ransohoff RM, Schafer D, Vincent A, Blachere NE, Bar-Or A. Neuroinflammation: Ways in Which the Immune System Affects the Brain. Neurotherapeutics : the journal of the American Society for Experimental NeuroTherapeutics. 2015; 12(4):896-909. [PubMed: 26306439]

108. Engelhardt B. The blood-central nervous system barriers actively control immune cell entry into the central nervous system. Current pharmaceutical design. 2008; 14(16):1555-1565. [PubMed: 18673197]

109. Block ML, Zecca L, Hong JS. Microglia-mediated neurotoxicity: uncovering the molecular mechanisms. Nat Rev Neurosci. 2007; 8(1):57-69. [PubMed: 17180163]

110. Schallner N, Ulbrich F, Engelstaedter H, et al. Isoflurane but not sevoflurane or desflurane aggravates injury to neurons in vitro and in vivo via p75NTR-NF-kB activation. Anesth Analg. 2014; 119(6):1429-1441. [PubMed: 25329094]

111. Lee YM, Song BC, Yeum KJ. Impact of Volatile Anesthetics on Oxidative Stress and Inflammation. BioMed research international. 2015; 2015:242709. [PubMed: 26101769]

112. Callaway JK, Wood C, Jenkins TA, Royse AG, Royse CF. Isoflurane in the presence or absence of surgery increases hippocampal cytokines associated with memory deficits and responses to brain injury in rats. Behavioural brain research. 2016; 303:44-52. [PubMed: 26784560]

113. Wu X, Lu Y, Dong Y, et al. The inhalation anesthetic isoflurane increases levels of proinflammatory TNF-alpha, IL-6, and IL-1beta. Neurobiology of aging. 2012; 33(7):13641378. [PubMed: 21190757]

114. Zhang L, Zhang J, Yang L, Dong Y, Zhang Y, Xie Z. Isoflurane and sevoflurane increase interleukin-6 levels through the nuclear factor-kappa B pathway in neuroglioma cells. British journal of anaesthesia. 2013; 110(Suppl 1):i82-91. [PubMed: 23604542]

115. Wagner M, Ryu YK, Smith SC, Patel P, Mintz CD. Review: effects of anesthetics on brain circuit formation. Journal of neurosurgical anesthesiology. 2014; 26(4):358-362. [PubMed: 25144504] 


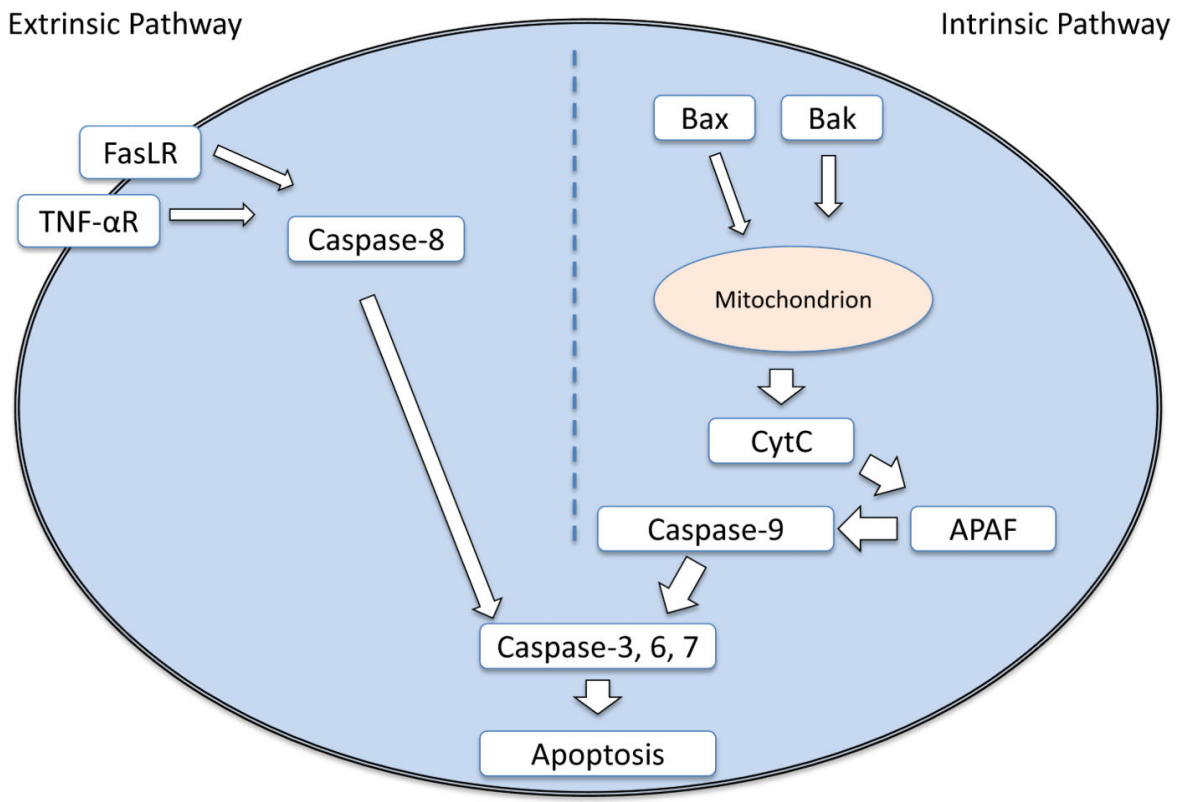

Fig. 1.

A Systematic Review of Pre-clinical Studies 


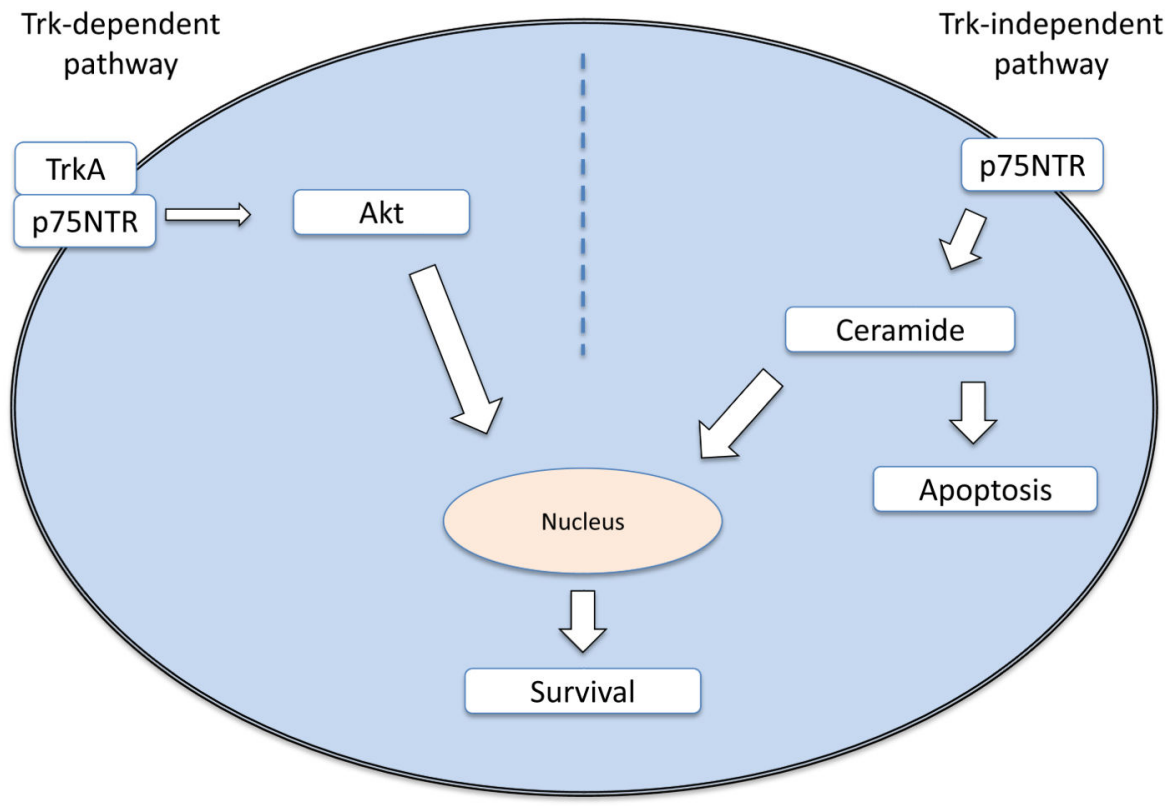

Fig. 2.

A Systematic Review of Pre-clinical Studies 


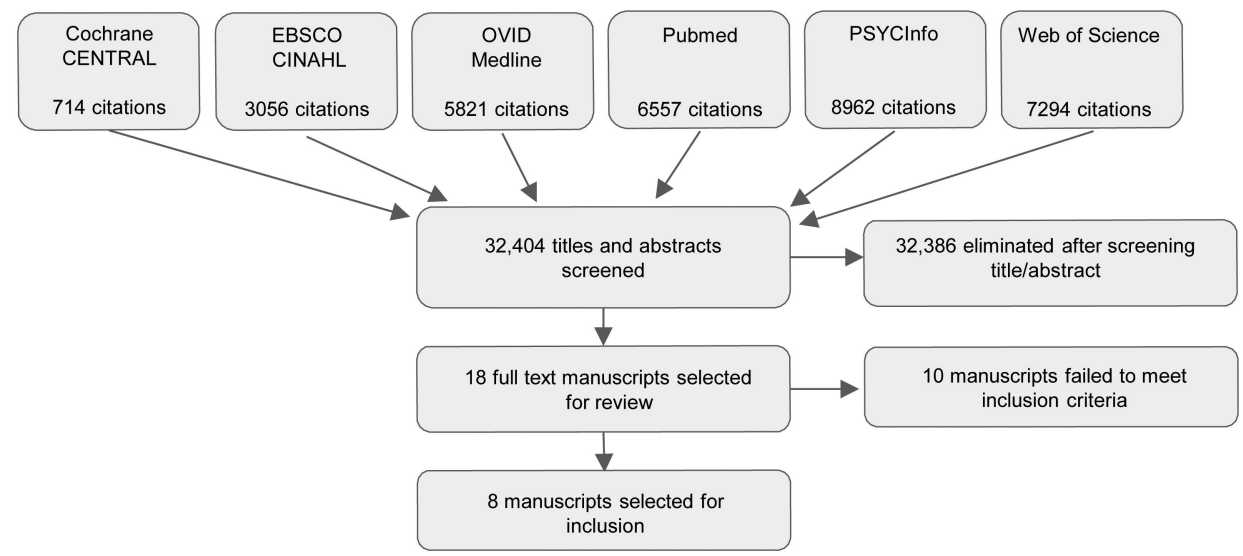

Fig. 3.

Physical Activity and Cognitive Development-A Meta-analysis

J Neurosurg Anesthesiol. Author manuscript; available in PMC 2017 October 01. 